\title{
Transcriptomic Analysis Reveals That Reactive Oxygen Species and Genes Encoding Lipid Transfer Protein Are Associated with Tobacco Hairy Root Growth and Branch Development
}

\author{
Jung-Hao Wang, ${ }^{1}$ Hsiao-Han Lin, ${ }^{1}$ Chi-Te Liu, ${ }^{2,3}$ Ta-Chung Lin, ${ }^{1}$ Li-yu Daisy Liu, ${ }^{4}$ and Kung-Ta Lee ${ }^{1}$ \\ ${ }^{1}$ Department of Biochemical Science and Technology, National Taiwan University, No. 1, Roosevelt Rd. Section 4, Taipei \\ 10617, Taiwan; ${ }^{2}$ Institute of Biotechnology, National Taiwan University, No. 81, Chang-Xing St., Taipei 10617, Taiwan; \\ ${ }^{3}$ Agricultural Biotechnology Research Center, Academia Sinica, No. 128, Academia Rd. Section 2, Nangang, Taipei 11529, \\ Taiwan; ${ }^{4}$ Department of Agronomy, National Taiwan University, No. 1
}

Submitted 17 December 2013. Accepted 24 February 2014.

\begin{abstract}
The hairy root, a specialized plant tissue that emerges from a cell transformed with transfer DNA (T-DNA) from Agrobacterium rhizogenes, can be used to study root biology and utilized in biotechnological applications. The rol genes are known to participate in the generation of hairy roots; however, the means by which the rol genes contribute to the initiation and the maintenance of hairy roots remains largely unknown. We demonstrated that tobacco hairy roots lacking either rolB or rolC exhibited fewer branch roots and lost their growth ability in long-term subculture. Additionally, a microarray analysis revealed that the expression of several genes encoding lipid transfer proteins (LTP) and reactive oxygen species (ROS)-related genes was significantly suppressed in $\mathrm{rolB}$ - or rolC-deficient hairy roots. We found that hairy root clones that exhibited greater branching expressed higher levels of RolB or RolC and the genes encoding LTP identified from the microarray. When hairy roots were compared with intact roots, the expression levels of LTP-encoding genes were dramatically different. In addition, ROS were present at lower levels in rolB- and rolC-deficient hairy roots. We therefore suggest that upregulating LTP and increasing the level of ROS is important for hairy root growth.
\end{abstract}

The hairy root is a specialized root tissue induced by translocation and expression of transfer DNA (T-DNA) from Agrobacterium rhizogenes in plant cells. The transformed tissue displays root-like characteristics and has many biotechnological advantages. For example, hairy roots grow rapidly with no requirement for exogenous phytohormones, can be transformed easily with other heterologous oligonucleotide fragments, and possess long-term morphological and genetic stability (Hamill et al. 1986; Sun et al. 1991). These features make cultured hairy roots a favored tissue for root biology studies, such as those involving legume-rhizobium symbiosis and nicotine biosynthesis regulation (Boisson-Dernier et al. 2001; DeBoer et al. 2011; Shoji et

Corresponding author: Kung-Ta Lee; E-mail: ktlee@ntu.edu.tw

* The $e$-Xtra logo stands for "electronic extra" and indicates that three supplementary tables and two supplementary figures are published online.

(C) 2014 The American Phytopathological Society al. 2010). Another advantage is that larger amounts of secondary metabolites accumulate in hairy roots versus cell suspension or callus culture (Bulgakov 2008; Zhou et al. 2011). Furthermore, hairy root biomass can be easily scaled up for production. Hairy root culture is, therefore, viewed as the most important secondary metabolite-producing system for the pharmaceutical industry (Baque et al. 2011; Ono and Tian 2011).

Despite their wide application, the use of hairy roots is limited because they can only be induced in certain plant species, most of which are dicots. Uncovering the mechanisms of hairy root formation and maintenance may allow the production of hairy roots in plants that have not been infected with $A$. rhizogenes.

Over the last three decades, many studies have aimed to reveal the mechanism of hairy root formation. In a transposonmutagenesis assay, four root loci ( $r o l)$ genes-rolA, rolB, rolC, and rolD-located on a T-DNA were identified as effective root-inducing genes (White et al. 1985). In isolation, rolA, rolB, and rolC have each been reported to be capable of inducing hairy root formation, although the effect is greater when more genes are involved (Spena et al. 1987). These results suggest that $\operatorname{rol} A, \operatorname{rolB}$, and $\operatorname{rolC}$ play crucial roles in hairy root development and in hormone regulation related to cell dedifferentiation, redifferentiation, and root structure maintenance. Many subsequent studies have focused on the functions of rolA, $\operatorname{rolB}$, and rolC in the plant cell. RolA contains a DNAbinding structure and is thought to be attached to the plasma membrane in the inactivated state (Levesque et al. 1988; Rigden and Carneiro 1999; Vilaine et al. 1998), suggesting that it is an inducible transcription factor. RolB has been suggested to be a membrane-bound tyrosine phosphatase (Filippini et al. 1996), which might translocate into the nucleus with the aid of 14-3-3 proteins (Moriuchi et al. 2004). RolC, a sucrose-inducible protein expressed in companion cells (Nilsson et al. 1996; Yokoyama et al. 1994), was initially considered to be a cytokinin enhancer (Estruch et al. 1991; Schmulling et al. 1988); however, subsequent studies disagreed with this result (Faiss et al. 1996; Nilsson et al. 1996). Additionally, the biochemical and cellular functions of rolA, rolB, and rolC have not yet been evaluated. RolD is the only Rol protein with a known functional role as an ornithine cyclodeaminase, which converts ornithine into proline (Trovato et al. 2001).

Although many reports have indicated that the rol genes are important in the induction of hairy root formation, little is 
known about how they stimulate and maintain hairy root growth. In this study, we first focused on how knockouts of each rol gene affected hairy root morphology by recording the growth of main roots or branch roots or both. Of the four genes, $\mathrm{rolB}$ and $\mathrm{rol} C$ were found to be more important for the induction of hairy root growth, including the promotion of branch root growth and the maintenance of hairy root architecture, activities that are in agreement with the roles of RolB and RolC proteins in long-term growth. We then compared the transcriptome of hairy roots infected by $\mathrm{rolB}$ - or $\mathrm{rolC}$-deficient $A$. rhizogenes with that of normal hairy roots that were infected by wild-type $A$. rhizogenes. We also compared the transcriptome of fast-growing normal hairy roots with that of intact tobacco roots. Combining the results of quantitative reverse transcription-polymerase chain reaction (qRT-PCR) and tissue staining, we suggest that hairy root growth and branch development are associated with reactive oxygen species (ROS) and genes encoding lipid transfer proteins (LTP).

\section{RESULTS}

\section{Generation of $A$. rhizogenes A4 clones} with rol gene deletions.

To clarify the effects of rol genes on plant root cells, each rol gene was deleted separately from $A$. rhizogenes using $c c d B$-based homologous recombination techniques (Schaefer et al. 1994), and the resulting mutants were designated as $\Delta \operatorname{rol} A, \Delta \operatorname{rolB}, \Delta \operatorname{rol} C$, and $\Delta$ rolD. These rol gene mutants were confirmed by Southern blot analysis (Supplementary Fig. S1A). When the growth rate of each mutant was compared with that of the wild-type (WT) strain in yeast extract broth (YEB) medium, WT A. rhizogenes A4 grew more slowly than its rol gene-deficient derivatives over a period of 12 to $24 \mathrm{~h}$. However, there was no significant difference among the five groups for incubations longer than $24 \mathrm{~h}$. Based on our results and those from other studies (Batra et al. 2004; Lee et al. 2007), we used 48-h cultures in the subsequent hairy root induction experiments.

\section{$\Delta r o l B$ and $\triangle r o l C A$. rhizogenes mutants}

have decreased hairy root induction ability.

To evaluate how rol genes affect the initiation of hairy roots, we observed leaf discs every day for three weeks after Agrobacterium infection to determine the day of first root emergence postinfection (DREPI). Meanwhile, we calculated the primary root number per leaf disc $(\mathrm{R} / \mathrm{L}$ ratio) at 21 days postinfection (dpi) (Swain et al. 2010). We defined induced hairy root tissue as a root-like tissue longer than $0.5 \mathrm{~cm}$. An earlier DREPI and a higher R/L ratio indicate superior hairy root induction ability.

Hairy roots began to emerge at $10 \mathrm{dpi}$ from WTA4-infected tobacco leaf discs, and by $21 \mathrm{dpi}$, more than $90 \%$ of the WTinfected leaf discs had at least one hairy root (Fig. 1A). A significantly delayed DREPI was observed in $\triangle \mathrm{rolB}$ - and $\Delta \mathrm{rolC}$ infected leaf discs compared with WT-infected discs $(P=1.1 \times$ $10^{-3}$ and $8.4 \times 10^{-6}$, respectively), but this delay was not observed in either $\Delta$ rolA- or $\Delta$ rolD-infected leaf discs $(P=0.72$ and 0.12 , respectively). $\Delta$ rolD-infected leaf discs showed a lower DREPI prior to but not after 16 dpi, showing no significant difference compared with WTA4-infected leaf discs. There was little or no difference in the percentage of $\Delta$ rolAinfected leaf discs showing hairy root emergence compared with that in WTA4-infected discs. Statistical analysis of the $\mathrm{R} / \mathrm{L}$ ratio at 21 dpi revealed significant differences between WTA4-infected leaf discs and either $\Delta$ rolB-infected leaf discs $\left(P=1.1 \times 10^{-3}\right)$ or $\Delta$ rol $C$-infected leaf discs $\left(P=8.4 \times 10^{-6}\right)$ but not $\triangle$ rolA- or $\Delta$ rolD-infected leaf discs $(P=0.19$ and 1.00 , respectively) (Fig. 1B). These results indicate that $\Delta r o l B$ - and $\Delta$ rolC-infected leaf discs had impaired hairy root initiation ability, whereas $\Delta$ rolA- or $\Delta$ rolD-infected leaf discs did not.

\section{Aberrant hairy roots induced by rol-deficient $A$. rhizogenes mutants.}

To understand how the rol genes affect hairy root growth, we established five groups of hairy roots by induction with wild-type $A$. rhizogenes and each rol-deficient $A$. rhizogenes clone; these groups were named HRWT, HR $\Delta$ rolA, HR $\Delta$ rolB, $\mathrm{HR} \Delta \mathrm{rol} C$, and HR $\Delta$ rolD. We then evaluated four parameters to determine the morphology at 18 days postsubculture: the main root length (MRL), branch root number (BRN), branch root density (BRD), and total branch root length (TBRL). MRL is defined as the length that the root grew from the root tip after subculture, BRN is the number of primary branch roots that emerged from the main root, BRD is the average number of branch roots per centimeter of main root, and TBRL is the sum of the root length of the whole hairy root culture, except for the main root. MRL and TBRL are used to evaluate rootgrowth ability of the original and new-forming root apical meristems, respectively. BRD and BRN describe lateral root emergence rate, which stands for new meristem formation. To
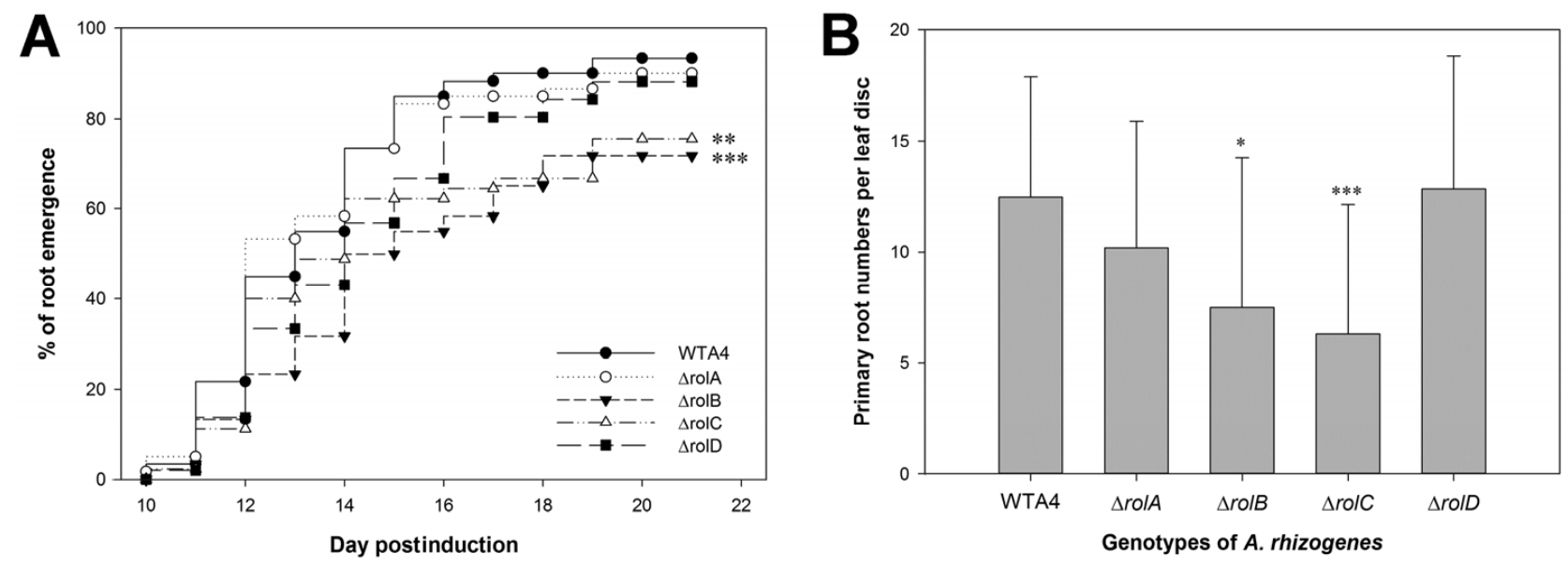

Fig. 1. Hairy root initiation ability of wild type and different rol-deficient Agrobacterium rhizogenes mutants. A, Day of first root emergence postinfection data. Hairy roots began to emerge at 12 days postinfection (dpi); by 21 dpi, more than $90 \%$ of the leaf discs in the WTA4-infected group showed hairy roots. The $\%$ of root emergence indicates the percentage of leaf discs with hairy roots. B, Number of hairy roots per leaf disc at 21 dpi. In total, $60,60,60,45$, and 52 replicates were performed for WTA4, $\Delta \operatorname{rolA}, \Delta \operatorname{rolB}, \Delta \operatorname{rolC}$, and $\Delta \operatorname{rol} D$, respectively. $(*$ indicates $P<0.05 ; * *, P<0.01 ;$ and $* * *,=P<0.001)$. 
select representative hairy root clones, all the hairy root clones of each group were sorted by their MRL, from the longest to the shortest, and one representative clone with an MRL in each of the first, 25th, 50th, 75th, and 100th percentiles was photographed. As shown in Figure 2, in which representative clones from the 1st to 100th percentile are arranged from left to right, $\mathrm{HR} \Delta$ rolB and HR $\Delta$ rolC clearly showed growth retardation.

We performed a permutation test using analysis of variance (ANOVA) with 1,000 replications to evaluate the differences among these hairy root groups, as statistical analyses revealed that none of the groups showed a normal distribution (Supplementary Fig. S2). The results of the MRL analysis (Fig. 3A) showed significant differences between HRWT and HR $\Delta$ rolC $\left(P=9.99 \times 10^{-4}\right)$ and between HRWT and HR $\Delta$ rolD $(P=9.99$ $\left.\times 10^{-3}\right)$. In contrast, there was a slight but not significant difference between HRWT and HR $\operatorname{rolA}\left(P=7.79 \times 10^{-2}\right)$ and between HRWT and HR $\Delta \operatorname{rolB}(P=0.125)$, indicating that all four of these genes contribute to the growth of the main root to different degrees. In the analyses of BRN (Fig. 3B), there was a significant difference between HRWT and HR $\Delta$ rolB $(P=$ $\left.7.99 \times 10^{-3}\right)$ and between HRWT and HRArolC $(P=9.99 \times$ $\left.10^{-4}\right)$; however, there was no significant difference between HRWT and either HR $\Delta$ rolA $(P=0.865)$ or $\operatorname{HR} \Delta \operatorname{rolD}(P=$ $0.549)$. To examine branch formation activity, we determined $\mathrm{BRD}$, the number of branch roots per centimeter of main root (Fig. 3C). We found a significant difference between HRWT and HRArolC $\left(P=9.99 \times 10^{-4}\right)$ and a slight difference between HRWT and HR $\Delta \operatorname{rolB}\left(P=7.89 \times 10^{-2}\right)$, with no significant difference between HRWT and either HR $\Delta \operatorname{rolA}(P=0.169)$ or HR $\Delta$ rolD $(P=0.387)$. In terms of TBRL, which was the other parameter used to estimate branch root architecture development (Fig. 3D), a significant difference was observed between
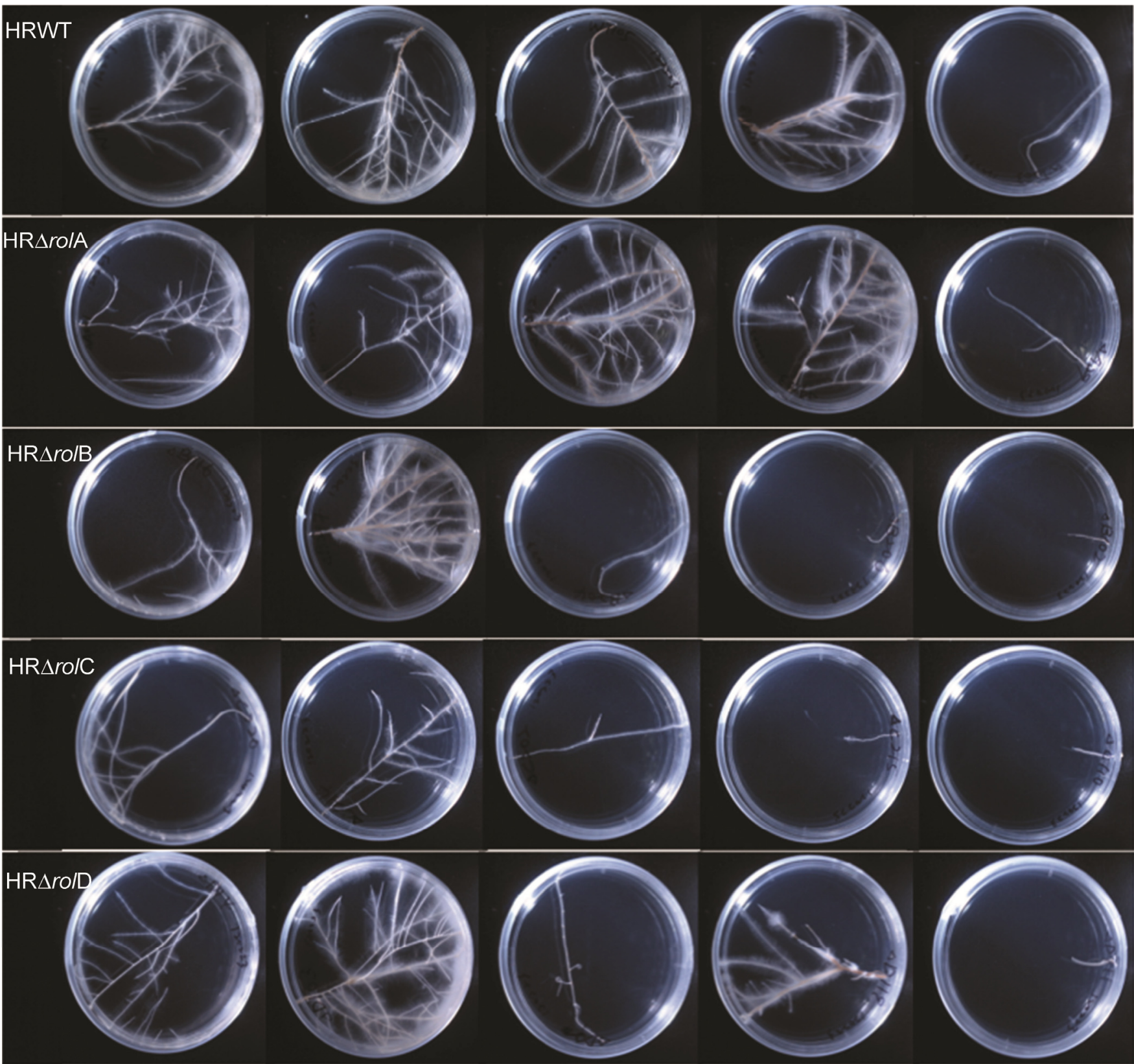

Fig. 2. Morphology of hairy roots at 18 days after subculture. Each hairy root was cut $1.5 \mathrm{~cm}$ from the root tip, and this segment was placed in fresh medium. After 18 days, all the clones were sorted and were photographed according to the main root length from the longest to the shortest. A representative clone (mutant or HRWT) with a main root length in each of the first, 25th, 50th, 75th, and 100th percentiles (arranged left to right) is shown. The number of independent clones examined was 84 for HRWT, 93 for HR $\Delta$ rolA, 65 for HR $\Delta$ rolB, 82 for HR $\Delta$ rolC, and 58 for HR $\Delta$ rolD. 
HRWT and HR $\Delta$ rolB $\left(P=4.39 \times 10^{-2}\right)$ and between HRWT and HR $\operatorname{rolC}\left(P=9.99 \times 10^{-4}\right)$, whereas there was a slight difference between HRWT and HR $\Delta$ rolA $\left(P=5.69 \times 10^{-2}\right)$ and between HRWT and HR $\Delta$ rolD $\left(P=5.39 \times 10^{-2}\right)$. Combining the MRL and TBRL results, all four of these genes can enhance both main root and branch root elongation to different degrees, but the BRN and BRD results showed that rolB and rolC are important for promoting branch formation and $\mathrm{rolC}$ is more effective than $\mathrm{rolB}$. In addition, after subculturing every two weeks for eight months, the clone survival rates (number of surviving clones to total clones) for HR $\Delta \mathrm{rolB}$ and $\mathrm{HR} \Delta \mathrm{rolC}$ were only 12.5 and $4.17 \%$, respectively, whereas those for HR $\Delta$ rolA and HRArolD were 87.5 and $95.8 \%$, respectively. This result indicates that $\mathrm{rolB}$ and $\mathrm{rolC}$ are essential for maintaining hairy root growth.

To confirm that plants lacking $\mathrm{rolB}$ or rolC exhibited decreased hairy root initiation and growth ability, we performed a complementation test in which tobacco was infected with complementary A. rhizogenes strains. The complemented rolB

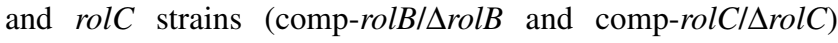
were constructed by transforming the $A$. rhizogenes knockout strain with a binary vector carrying the corresponding rol gene driven by its native promoter. The complemented hairy roots simultaneously harbored the original T-DNA region from the Ri plasmid and plasmid T-DNA harboring the comp-rol gene. The results showed that hairy root initiation and growth ability were only partially recovered (data not shown). These results suggest that the complementation test has drawbacks. Every complemented hairy root clone may differ in T-DNA number and insertion site. Additionally, the ratio of T-DNAs from the Ri plasmid to T-DNAs from the binary vector may vary, which results in a complicated and uncontrollable situation. To avoid this complexity, we increased the sample size in each group to ensure that the phenomenon observed generally existed in a group and was most likely due to the lack of $\mathrm{rolB}$ and $\mathrm{rolC}$.

Taken together, these data show that rolC- and rolD-deficient hairy roots exhibited greater growth retardation in the main root, whereas rolB- or rolC-deficient hairy roots exhibited de-
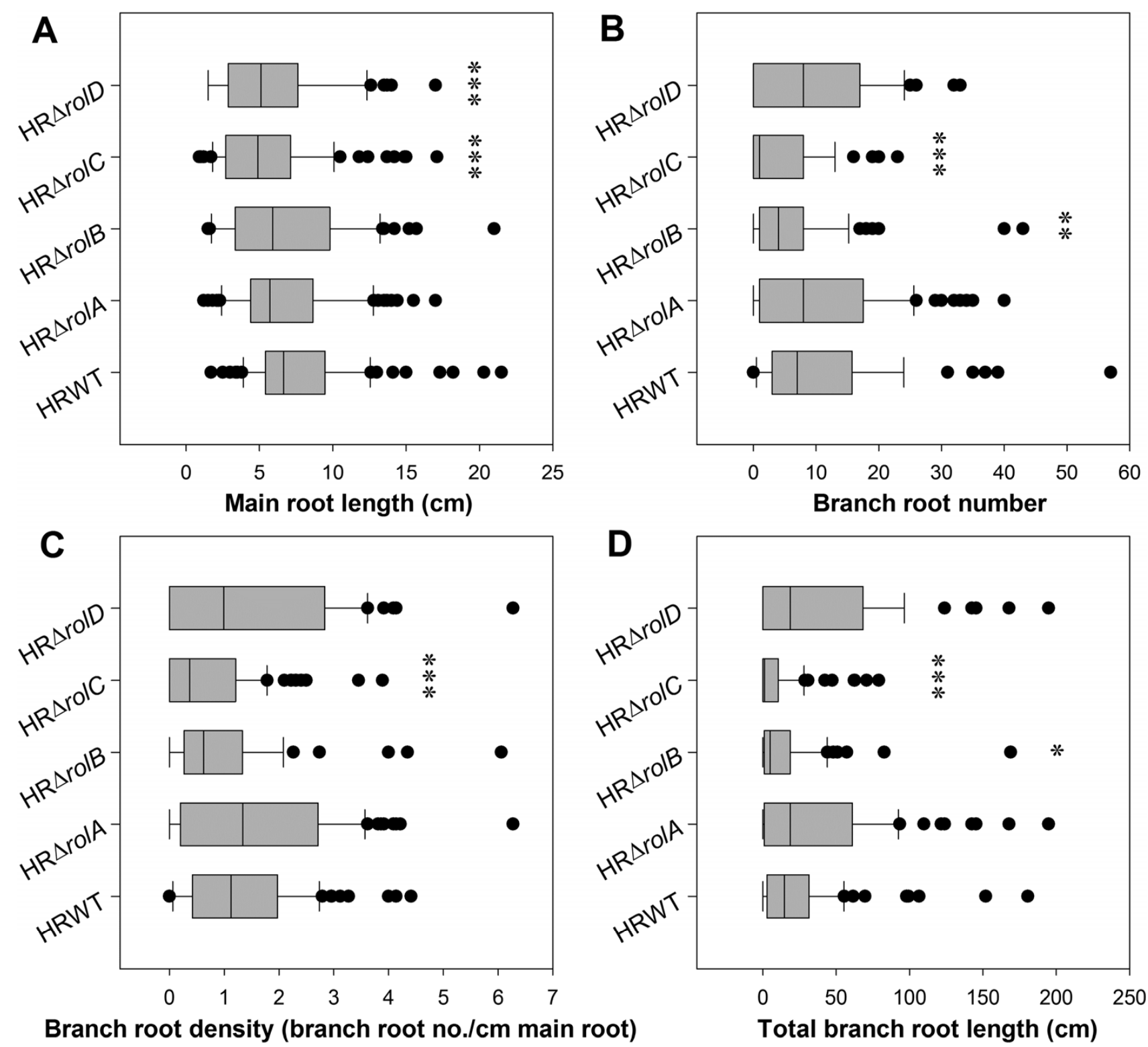

Fig. 3. Box plot analysis of hairy root architecture. A $1.5-\mathrm{cm}$ section of each hairy root from the root tip was removed and subcultured. At 18 days postsubculture, the following parameters were measured: $\mathbf{A}$, main root length, $\mathbf{B}$, number of branch roots, $\mathbf{C}$, total branch root length, and $\mathbf{D}$, branch root density. Significant differences were determined by permutation test using analysis of variance with 1,000 replications against HRWT. (* $P<0.05$, $* * P<$ 0.01 , *** $P<0.001$ ). 
creased hairy root initiation, branch root formation and elongation, and long-term growth ability.

\section{Microarray data analysis.}

To further elucidate how $\mathrm{rolB}$ and $\mathrm{rolC}$ enhance hairy root branching and growth, we compared the transcriptomic differences between HRWT and HR $\Delta$ rolB and between HRWT and HR $\Delta$ rolC. We performed two-channel dye-swap microarray assays with two biological replicates. To reduce the variations between different clones, we pooled 24 hairy root clones with the same tissue weight and extracted RNA for the microarray hybridization. Based on the results of statistical analysis, the pooled samples had similar medians and similar variation in the four parameters, and therefore, differences in the transcriptomes as shown in the microarray data were due to the gene deficiencies rather than phenotypic variations. Genes consistently showing greater than a twofold difference in expression are listed in Supplementary Table S1. Compared with HRWT, there were six genes in HR $\Delta$ rolB that showed a more than twofold increase in expression and 242 genes that were decreased to less than 0.5 -fold; the corresponding numbers for HR $\Delta$ rolC were 42 and 208, respectively.

Gene Ontology (GO) enrichment analysis was performed using agriGO with the default settings (Du et al. 2010). In to-

Table 1. Gene Ontology results for transcripts downregulated in HR $\Delta$ rolB as compared with HRWT (hairy roots induced with wild-type Agrobacterium rhizogenes) $(P<0.05)$

\begin{tabular}{|c|c|c|}
\hline Description & $P$ value & $\mathbf{F D R}^{\mathbf{z}}$ \\
\hline Lipid transport & $1.8 \times 10^{-15}$ & $3.0 \times 10^{-13}$ \\
\hline Lipid localization & $3.1 \times 10^{-15}$ & $3.0 \times 10^{-13}$ \\
\hline Lipid binding & $2.8 \times 10^{-11}$ & $2.1 \times 10^{-9}$ \\
\hline Macromolecule localization & $1.7 \times 10^{-6}$ & $1.1 \times 10^{-4}$ \\
\hline Response to wounding & $2.8 \times 10^{-6}$ & $1.4 \times 10^{-4}$ \\
\hline $\begin{array}{l}\text { Hydrolase activity, hydrolyzing } O \text {-glycosyl } \\
\text { compounds }\end{array}$ & $7.3 \times 10^{-6}$ & $2.8 \times 10^{-4}$ \\
\hline Hydrolase activity, acting on glycosyl bonds & $2.6 \times 10^{-5}$ & $6.6 \times 10^{-4}$ \\
\hline Endomembrane system & $3.3 \times 10^{-5}$ & $1.8 \times 10^{-3}$ \\
\hline Response to chemical stimulus & $1.8 \times 10^{-4}$ & $6.8 \times 10^{-3}$ \\
\hline $\begin{array}{l}\text { Oxidoreductase activity, acting on peroxide } \\
\text { as an acceptor }\end{array}$ & $2.5 \times 10^{-4}$ & $3.8 \times 10^{-3}$ \\
\hline Peroxidase activity & $2.5 \times 10^{-4}$ & $3.8 \times 10^{-3}$ \\
\hline Response to stimulus & $2.6 \times 10^{-4}$ & $8.0 \times 10^{-3}$ \\
\hline Response to stress & $2.9 \times 10^{-4}$ & $8.0 \times 10^{-3}$ \\
\hline Response to external stimulus & $4.0 \times 10^{-4}$ & $9.5 \times 10^{-3}$ \\
\hline Electron carrier activity & $5.0 \times 10^{-4}$ & $6.4 \times 10^{-3}$ \\
\hline Antioxidant activity & $8.9 \times 10^{-4}$ & $9.7 \times 10^{-3}$ \\
\hline Extracellular region & $9.6 \times 10^{-4}$ & $2.6 \times 10^{-2}$ \\
\hline Oxidoreductase activity & $1.0 \times 10^{-3}$ & $9.7 \times 10^{-3}$ \\
\hline Lyase activity & $1.3 \times 10^{-3}$ & $1.1 \times 10^{-2}$ \\
\hline Transport & $1.5 \times 10^{-3}$ & $3.1 \times 10^{-2}$ \\
\hline Establishment of localization & $1.6 \times 10^{-3}$ & $3.1 \times 10^{-2}$ \\
\hline Cation binding & $2.0 \times 10^{-3}$ & $1.4 \times 10^{-2}$ \\
\hline Ion binding & $2.0 \times 10^{-3}$ & $1.4 \times 10^{-2}$ \\
\hline $\begin{array}{l}\text { Cellular amino acid derivative biosynthetic } \\
\text { process }\end{array}$ & $2.1 \times 10^{-3}$ & $3.4 \times 10^{-2}$ \\
\hline Localization & $2.2 \times 10^{-3}$ & $3.4 \times 10^{-2}$ \\
\hline Response to endogenous stimulus & $2.3 \times 10^{-3}$ & $3.4 \times 10^{-2}$ \\
\hline $\begin{array}{l}\text { Oxidoreductase activity, acting on the } \mathrm{CH}-\mathrm{OH} \\
\text { group of donors, NAD, or NADP as an } \\
\text { acceptor }\end{array}$ & $2.6 \times 10^{-3}$ & $1.7 \times 10^{-2}$ \\
\hline Response to hormone stimulus & $3.4 \times 10^{-3}$ & $4.4 \times 10^{-2}$ \\
\hline Response to ethylene stimulus & $3.5 \times 10^{-3}$ & $4.4 \times 10^{-2}$ \\
\hline $\begin{array}{l}\text { Oxidoreductase activity, acting on the } \mathrm{CH}-\mathrm{OH} \\
\text { group of donors }\end{array}$ & $4.5 \times 10^{-3}$ & $2.7 \times 10^{-2}$ \\
\hline $\begin{array}{l}\text { Cellular amino acid derivative metabolic } \\
\text { process }\end{array}$ & $6.7 \times 10^{-3}$ & $8.0 \times 10^{-2}$ \\
\hline Response to organic substances & $1.3 \times 10^{-2}$ & $1.4 \times 10^{-1}$ \\
\hline Carbohydrate metabolic process & $2.3 \times 10^{-2}$ & $2.4 \times 10^{-1}$ \\
\hline Transition metal ion binding & $3.0 \times 10^{-2}$ & $1.6 \times 10^{-1}$ \\
\hline Metal ion binding & $3.1 \times 10^{-2}$ & $1.6 \times 10^{-1}$ \\
\hline
\end{tabular}

${ }^{\mathrm{z}} \mathrm{FDR}=$ false discovery rate. tal, 101 of the 242 genes that were downregulated in HR $\Delta$ rolB and 88 of the 208 genes that were downregulated in HR $\Delta$ rolC were annotated. The GO terms with a $P$ value less than 0.05 are shown in Tables 1 and 2. Interestingly, the gene sets showing lower expression levels in HR $\Delta$ rolB and HR $\Delta$ rolC were quite similar, demonstrating that branch root density and hairy root growth are regulated by these genes. The gene sets that showed lower expression levels in HR $\Delta$ rolB and HR $\Delta$ rolC mainly contained LTP genes as well as genes annotated with the GO terms "response to wounding" and "responses to chemical stimuli." Genes annotated with the GO terms "responses to wounding" and "responses to chemical stimuli" have been shown to generate ROS (Bhattacharjee 2012). Moreover, based on molecular function analysis, HR $\Delta$ rolB and HR $\Delta$ rolC had decreased oxidoreductase activity, indicating that the ROS levels were altered. Additionally, genes involved in ion balance, carbon and nitrogen metabolism, and molecular transport and localization were downregulated in HR $\Delta$ rolB and HR $\Delta$ rolC roots compared with normal hairy roots. We hypothesize that lipid signals, ROS, ions, metabolism, and molecular transport and localization are important for promoting branch growth and maintaining growth activities in hairy roots.

To confirm the above-mentioned hypothesis, we compared the transcriptomes between a fast-growing HRWT and tobacco intact roots by dye-swap two-channel microarray. The dominant GO enrichment terms $\left(P<10^{-6}\right)$ found in hairy roots are listed in Table 3 . We found that hairy roots expressed more transcripts related to cell replication, including genes involved in the cell cycle, cell-wall biogenesis, cell-component biogenesis, DNA replication, and carbon and nitrogen metabolism.

Table 2. Gene Ontology results for transcripts downregulated in HR $\Delta$ rolC compared with HRWT (hairy roots induced with wild-type Agrobacterium rhizogenes $)(P<0.05)$

\begin{tabular}{|c|c|c|}
\hline Description & $P$ value & $\mathbf{F D R}^{\mathbf{z}}$ \\
\hline Lipid transport & $6.0 \times 10^{-12}$ & $6.4 \times 10^{-10}$ \\
\hline Lipid localization & $9.1 \times 10^{-12}$ & $6.4 \times 10^{-10}$ \\
\hline $\begin{array}{l}\text { Hydrolase activity, hydrolyzing } O \text {-glycosyl } \\
\text { compounds }\end{array}$ & $4.4 \times 10^{-10}$ & $2.6 \times 10^{-8}$ \\
\hline Hydrolase activity, acting on glycosyl bonds & $3.0 \times 10^{-9}$ & $8.7 \times 10^{-8}$ \\
\hline Lipid binding & $2.1 \times 10^{-7}$ & $4.0 \times 10^{-6}$ \\
\hline Extracellular region & $8.0 \times 10^{-6}$ & $8.4 \times 10^{-4}$ \\
\hline Endomembrane system & $1.8 \times 10^{-5}$ & $9.5 \times 10^{-4}$ \\
\hline Macromolecule localization & $5.2 \times 10^{-5}$ & $2.4 \times 10^{-3}$ \\
\hline Transport & $1.4 \times 10^{-4}$ & $4.1 \times 10^{-3}$ \\
\hline Establishment of localization & $1.5 \times 10^{-4}$ & $4.1 \times 10^{-3}$ \\
\hline Response to chemical stimulus & $1.9 \times 10^{-4}$ & $4.1 \times 10^{-3}$ \\
\hline Localization & $2.2 \times 10^{-4}$ & $4.1 \times 10^{-3}$ \\
\hline Response to stimulus & $2.3 \times 10^{-4}$ & $4.1 \times 10^{-3}$ \\
\hline Copper ion binding & $2.7 \times 10^{-4}$ & $4.0 \times 10^{-3}$ \\
\hline Apoplast & $4.4 \times 10^{-4}$ & $1.6 \times 10^{-2}$ \\
\hline Response to wounding & $5.2 \times 10^{-4}$ & $8.1 \times 10^{-3}$ \\
\hline Carbohydrate metabolic process & $1.1 \times 10^{-3}$ & $1.5 \times 10^{-2}$ \\
\hline Ion binding & $1.5 \times 10^{-3}$ & $1.4 \times 10^{-2}$ \\
\hline Cation binding & $1.5 \times 10^{-3}$ & $1.4 \times 10^{-2}$ \\
\hline Chloroplast thylakoid & $4.9 \times 10^{-3}$ & $8.8 \times 10^{-2}$ \\
\hline Plastid thylakoid & $4.9 \times 10^{-3}$ & $8.8 \times 10^{-2}$ \\
\hline Organelle subcompartment & $5.0 \times 10^{-3}$ & $8.8 \times 10^{-2}$ \\
\hline Thylakoid part & $6.1 \times 10^{-3}$ & $9.2 \times 10^{-2}$ \\
\hline Ion transport & $6.3 \times 10^{-3}$ & $7.5 \times 10^{-2}$ \\
\hline Response to metal ion & $6.4 \times 10^{-3}$ & $7.5 \times 10^{-2}$ \\
\hline Response to inorganic substance & $9.5 \times 10^{-3}$ & $1.0 \times 10^{-1}$ \\
\hline Lyase activity & $1.0 \times 10^{-2}$ & $8.6 \times 10^{-2}$ \\
\hline Response to hormone stimulus & $1.1 \times 10^{-2}$ & $1.1 \times 10^{-1}$ \\
\hline Response to stress & $1.2 \times 10^{-2}$ & $1.1 \times 10^{-1}$ \\
\hline Response to external stimulus & $1.2 \times 10^{-2}$ & $1.1 \times 10^{-1}$ \\
\hline Response to endogenous stimulus & $1.8 \times 10^{-2}$ & $1.5 \times 10^{-1}$ \\
\hline Thylakoid & $2.0 \times 10^{-2}$ & $2.6 \times 10^{-1}$ \\
\hline Metal ion binding & $2.9 \times 10^{-2}$ & $2.1 \times 10^{-1}$ \\
\hline Transition metal ion binding & $3.2 \times 10^{-2}$ & $2.1 \times 10^{-1}$ \\
\hline
\end{tabular}

${ }^{\mathrm{z}} \mathrm{FDR}=$ false discovery rate. 
These findings are in agreement with the finding that hairy roots grew much faster than intact roots. We also found that the ROS-related transcripts and genes encoding LTP were upregulated in HRWT as compared with tobacco intact roots, and this result is consistent with the finding that HR $\Delta$ rolB and HR $\Delta$ rolC showed growth retardation and had lower LTP and ROS-related gene expression. These results strengthen the hypothesis that LTP and ROS-related genes play important roles in hairy root development. We confirm and discuss the relationship among hairy root growth, LTP, and ROS below.

\section{The expression levels of genes encoding LTP were related to hairy root growth.}

There have been few indications that genes encoding LTP are essential for hairy root growth and branch development. We first confirmed that the expression of genes encoding LTP in HRWT, $\mathrm{HR} \Delta \mathrm{rolB}$, and rolC is consistent with the results of the microarray (data not shown). Because HR $\Delta$ rolB and rolC showed de-

Table 3. Gene Ontology results for transcripts upregulated in HRWT (hairy roots induced with wild-type Agrobacterium rhizogenes) compared with tobacco intact roots $\left(P<10^{-5}\right)$

\begin{tabular}{lcc}
\hline Description & $\boldsymbol{P}$ value & FDR $^{\mathbf{Z}}$ \\
\hline Cell cycle & $6.2 \times 10^{-11}$ & $1.3 \times 10^{-7}$ \\
Lipid localization & $2.7 \times 10^{-9}$ & $2.8 \times 10^{-6}$ \\
Hydrolase activity, acting on glycosyl bonds & $2.9 \times 10^{-9}$ & $1.8 \times 10^{-6}$ \\
Lipid transport & $7.5 \times 10^{-9}$ & $5.2 \times 10^{-6}$ \\
Cell cycle process & $1.6 \times 10^{-8}$ & $8.1 \times 10^{-6}$ \\
Endomembrane system & $2.1 \times 10^{-8}$ & $6.7 \times 10^{-6}$ \\
Plant-type cell wall & $4.2 \times 10^{-8}$ & $6.7 \times 10^{-6}$ \\
External encapsulating structure & $4.7 \times 10^{-8}$ & $6.7 \times 10^{-6}$ \\
Hydrolase activity, hydrolyzing $O$-glycosyl & $8.6 \times 10^{-8}$ & $2.6 \times 10^{-5}$ \\
compounds & & \\
Cell wall & $2.2 \times 10^{-7}$ & $2.3 \times 10^{-5}$ \\
Antioxidant activity & $3.1 \times 10^{-7}$ & $6.1 \times 10^{-5}$ \\
Intracellular non-membrane-bounded organelle & $6.8 \times 10^{-7}$ & $4.8 \times 10^{-5}$ \\
Non-membrane bounded organelle & $6.8 \times 10^{-7}$ & $4.8 \times 10^{-5}$ \\
Chromosomal part & $8.0 \times 10^{-7}$ & $4.9 \times 10^{-5}$ \\
Regulation of cell cycle & $1.3 \times 10^{-6}$ & $5.3 \times 10^{-4}$ \\
Enzyme regulator activity & $1.5 \times 10^{-6}$ & $1.8 \times 10^{-4}$ \\
Xyloglucan:xyloglucosyl transferase activity & $1.5 \times 10^{-6}$ & $1.8 \times 10^{-4}$ \\
Carboxylesterase activity & $1.8 \times 10^{-6}$ & $1.8 \times 10^{-4}$ \\
Protein-DNA complex & $3.0 \times 10^{-6}$ & $1.6 \times 10^{-4}$ \\
Pectinesterase activity & $9.5 \times 10^{-6}$ & $8.2 \times 10^{-4}$ \\
Cytokineses during cell cycle & $9.7 \times 10^{-6}$ & $3.3 \times 10^{-3}$ \\
\hline
\end{tabular}

${ }^{\mathrm{z}} \mathrm{FDR}=$ false discovery rate. creased expression of genes encoding LTP and branch growth, we questioned whether branch development is related to LTP. We selected three HRWT clones with different BRD values and measured the expression of root growth promoting-rolB and rolC genes and LTP genes by qRT-PCR. As shown in Table 4, hairy roots with higher BRD showed increased LTP, rolB, and rol $C$ gene expression. Additionally, the expression levels of rolB and rolC showed a highly positive correlation (correlation coefficient $=0.988$ ), suggesting that the T-DNA integration locus determines the expression levels of the $\mathrm{rolB}$ and $\mathrm{rolC}$ genes. These data strongly suggest that $\mathrm{rolB}$, rolC, and genes encoding LTP promote branch development. We then compared the expression levels of genes encoding LTP in tobacco roots and leaves (Table 4). We discovered that the expression profiles of these LTP genes in tobacco roots and leaves were dramatically different from the profile in hairy roots, indicating that genes encoding LTP are expressed differently in hairy root tissues compared with normal tissue. This evidence indicates that hairy root growth and branch development are related to regulate the expression of genes encoding LTP.

\section{ROS accumulate in hairy roots but decrease when either rolB or rolC is knocked out.}

ROS have been considered as side products in growing tissue, although they have recently been considered as growth regulators in roots (Tsukagoshi et al. 2010). Previous research indicated that ROS levels were dramatically decreased in both Cauliflower mosaic virus (CaMV) 35S-rolB- and CaMV35SrolC-transformed cell suspension cultures (Bulgakov et al. 2008, 2012), but, these results conflict with our microarray data. To examine how ROS are regulated in HRWT, HR $\Delta$ rolB, and HR $\Delta$ rolC, we measured in situ ROS levels by staining with chloromethyl-2',7'-dichlorodihydrofluorescein diacetate $\left(\mathrm{CM}-\mathrm{H}_{2} \mathrm{DCFDA}\right)$. We observed that, in both the main roots and branch roots, ROS levels were much higher in HRWT than in HR $\Delta$ rolB or HR $\Delta$ rolC (Fig. 4). These results are consistent with the microarray results, indicating that HR $\Delta$ rolB and HR $\Delta$ rolC produce less ROS compared with HRWT. We hypothesize that the expression of $\mathrm{rolB}$ and $\mathrm{rolC}$ in different tissue types or the use of different promoters caused the differences in ROS levels. Further studies should be performed to clarify the relationship between hairy root growth and ROS accumulation.

Based on the findings, we concluded the hairy root growth and branch development promoted by $A$. rhizogenes rolB and

Table 4. The expression levels of rolB and rolC and lipid transfer proteins in hairy root clones with different branch root densities (BRD) ${ }^{\mathrm{Z}}$

\begin{tabular}{|c|c|c|c|c|c|}
\hline \multirow[b]{2}{*}{$\begin{array}{l}\text { Gene name or NCBI } \\
\text { accession number }\end{array}$} & \multicolumn{3}{|c|}{ HRWT } & \multirow[b]{2}{*}{ Tobacco root } & \multirow[b]{2}{*}{ Tobacco leaf } \\
\hline & $\begin{array}{c}\text { Clone 115 } \\
\text { BRD }=2.30 \pm \mathbf{0 . 6 0 a}\end{array}$ & $\begin{array}{c}\text { Clone } 87 \\
\text { BRD }=\mathbf{3 . 0 6} \pm \mathbf{0 . 2 0 a}\end{array}$ & $\begin{array}{c}\text { Clone 106 } \\
\text { BRD }=\mathbf{3 . 7 9} \pm \mathbf{0 . 0 7 b}\end{array}$ & & \\
\hline rolB & $0.00374 \pm 0.00065 a$ & $0.0139 \pm 0.0013 b$ & $0.0197 \pm 0.0068 b$ & & \\
\hline rolc & $0.464 \pm 0.196 \mathrm{a}$ & $1.25 \pm 0.17 \mathrm{~b}$ & $2.05 \pm 0.31 \mathrm{c}$ & & \\
\hline BQ842876 & $3.24 \pm 1.36 \mathrm{a}$ & $4.88 \pm 0.19 \mathrm{a}$ & $7.74 \pm 2.00 \mathrm{~b}$ & $0.951 \pm 0.106$ & $0.990 \pm 0.075$ \\
\hline EH618856/AB041519 & $8.40 \pm 2.98 \mathrm{a}$ & $12.3 \pm 1.35 \mathrm{ab}$ & $16.7 \pm 3.4 b$ & $0.954 \pm 0.045$ & $1.02 \pm 0.06$ \\
\hline EB443656 & $0.00680 \pm 0.00596 a$ & $0.0134 \pm 0.0036 a$ & $0.0378 \pm 0.0137 b$ & $1.06 \pm 0.02$ & $1.20 \pm 0.05$ \\
\hline EB450585 & $4.78 \pm 0.84 a$ & $8.56 \pm 3.05 a$ & $15.0 \pm 3.6 b$ & $0.909 \pm 0.006$ & $1.07 \pm 0.04$ \\
\hline D86629 & $4.86 \pm 0.71 \mathrm{a}$ & $8.79 \pm 3.25 \mathrm{a}$ & $14.5 \pm 2.5 b$ & $0.925 \pm 0.008$ & $1.10 \pm 0.06$ \\
\hline DV157577 & $0.0336 \pm 0.0120$ & $0.0679 \pm 0.0212$ & $0.106 \pm 0.047$ & $1.05 \pm 0.02$ & $1.17 \pm 0.04$ \\
\hline BQ842956 & $0.0340 \pm 0.0095 \mathrm{a}$ & $0.0581 \pm 0.0220 \mathrm{ab}$ & $0.0807 \pm 0.0190 \mathrm{~b}$ & $1.05 \pm 0.02$ & $1.18 \pm 0.05$ \\
\hline AF043554 & $1.02 \pm 0.16 \mathrm{a}$ & $2.41 \pm 1.18 \mathrm{ab}$ & $3.62 \pm 0.61 b$ & $0.950 \pm 0.014$ & $1.09 \pm 0.03$ \\
\hline AB035125 & $0.141 \pm 0.030 \mathrm{a}$ & $0.637 \pm 0.316 b$ & $1.17 \pm 0.20 \mathrm{c}$ & $1.00 \pm 0.06$ & $1.22 \pm 0.00$ \\
\hline DW003388 & $0.142 \pm 0.100$ & $0.324 \pm 0.160$ & $0.418 \pm 0.098$ & $1.02 \pm 0.03$ & $1.20 \pm 0.01$ \\
\hline FG191218 & $0.0384 \pm 0.0117 \mathrm{a}$ & $0.0957 \pm 0.0266 b$ & $0.147 \pm 0.036 b$ & $1.12 \pm 0.01$ & $1.02 \pm 0.05$ \\
\hline FG137954 & $0.111 \pm 0.018 \mathrm{a}$ & $0.204 \pm 0.049 \mathrm{a}$ & $0.453 \pm 0.070 b$ & $1.05 \pm 0.02$ & $1.03 \pm 0.05$ \\
\hline
\end{tabular}

${ }^{\mathrm{z}}$ HRWT $=$ hairy roots induced with wild-type Agrobacterium rhizogenes; NCBI = National Center for Biotechnology Information. The relative expression level of each gene is standardized with that of TAC-9 (tobacco ACTIN-9). The statistical analysis was performed by one-way analysis of variance, and significant differences were analyzed by Fisher's least significant difference. $P<0.05$. 
rolC genes are associated with altering the expression of plant genes encoding LTP and with ROS.

\section{DISCUSSION}

Over the past two decades, functional studies of the rol genes have been performed using transgenic plants, cell suspensions, and callus cultures, but seldom using hairy roots (Casanova et al. 2005). Because the rol genes are "root loci" genes and they encode proteins causing hairy root disease, we chose hairy root tissue to characterize the functions of the rol genes. Here, we found that $A$. rhizogenes lacking rolB or rolC showed decreased hairy root initiation ability (Fig. 1) and that hairy roots lacking $\mathrm{rolB}$ or $\mathrm{rolC}$ showed decreased numbers of branch roots and impaired elongation (Fig. 2). Moreover, rolB and rolC were important for hairy root survival in long-term subculture. These data suggest that $\mathrm{rolB}$ and rolC play crucial roles in regulating hairy root meristem activities, including enhancing branch formation and promoting branch root elongation. In contrast, rolA and rolD had minor effects on root initiation, branch root growth, and growth in long-term subculture. To our knowledge, this is the first study examining how rol genes function in hairy root branch development and longterm maintenance.

Compared with non-infected roots, hairy roots showed increases in various growth parameters, including growth rate, branch root number, and long-term maintenance of growth. These growth parameters were decreased in HR $\Delta$ rolB and $\mathrm{HR} \Delta$ rolC. Based on the microarray analysis, HR $\Delta$ rolB and HR $\Delta$ rolC have similar transcriptomic profiles, which include genes encoding LTP, ROS-related genes, and genes related to cellular metabolism and ion balance (Table 1 and 2). These data are consistent with the finding that $\mathrm{rolB}$ and $\mathrm{rolC}$ have similar effects on hairy roots. However, they are not redundant genes because they could not complement each other with respect to growth promotion (Fig. 2). Previous studies have also shown that the expression of $\mathrm{rolB}$ is induced by auxin and auxin-independent transcription factors, whereas the expression of rolC is induced by sucrose (DePaolis et al. 1996; Filetici et al. 1997). In addition, their sites of expression are different. The rolB promoter is activated in the root apical meristem and lateral primordia, whereas the rolC promoter is activated in the root apical meristem and phloem tissue (Maurel et al. 1994). These observations support the idea that $\mathrm{rolB}$ and $\mathrm{rolC}$ are sepa- rately regulated in roots and act synergistically to induce hairy root branch development and to maintain hairy root architecture.

Microarray analysis suggested that the biological function of rolB and rolC is linked to that of LTP, which might function together to promote hairy root growth. qRT-PCR results showed that branch root densities and LTP expression levels were highly positively correlated (Table 4). Tobacco leaves and roots have similar LTP gene expression profiles. However, in hairy roots, these genes are either dramatically upregulated or downregulated (Table 4). Genes encoding LTP are abundant in the plant kingdom, and the functions of most of them are known. In this study, genes were classified as LTP based on sequence annotations, and we found that regulating the expression levels of these LTP genes is important for mediating hairy root growth. In addition to our findings, there are several lines of evidence implying that the expression of RolB and LTP are related. First, LTP translocate lipid molecules inter- or intracellularly and lipids play a key role in determining cell differentiation, proliferation, and tissue development (Kader 1996). The cellular functions of these lipids overlap with those of RolB. Second, both RolB and LTP are expressed in the same regions of the root, including the apical meristem and branch root primordia (Capone et al. 1991; Thoma et al. 1994). Third, RolB is an auxin-inducible protein, and rolB-transformed cells show enhanced auxin perception (Maurel et al. 1994). In addition, LTP respond to auxin treatment in both hyacinth and rice plants (EMBL database and RiceXPro database). Therefore, we hypothesized that RolB enhances auxin signals, stimulating LTP expression. Here, we propose that RolB, RolC, and LTP may act synergistically or sequentially to regulate the fate of the hairy root, although the molecular mechanisms remain to be elucidated.

ROS are not merely side products of cell growth but key factors for modulating root differentiation and proliferation (Tsukagoshi et al. 2010). The means by which ROS are produced by cellular enzymes and the mechanism by which the plant cell regulates its ROS levels to influence growth are still unknown. Our microarray results showed that the expression of ROS-related genes was lower in HR $\Delta$ rolB and HR $\Delta$ rolC than in HRWT, and these results were further confirmed by fluorescence staining (Fig. 4). HR $\Delta$ rolB and HRWT had a similar MRL, but HR $\Delta$ rolB had lower ROS levels in the main root. This finding excludes the possibility that hairy root elongation contributes to ROS accumulation, although it suggests that RolB regulates cellular ROS levels to promote cell differ-
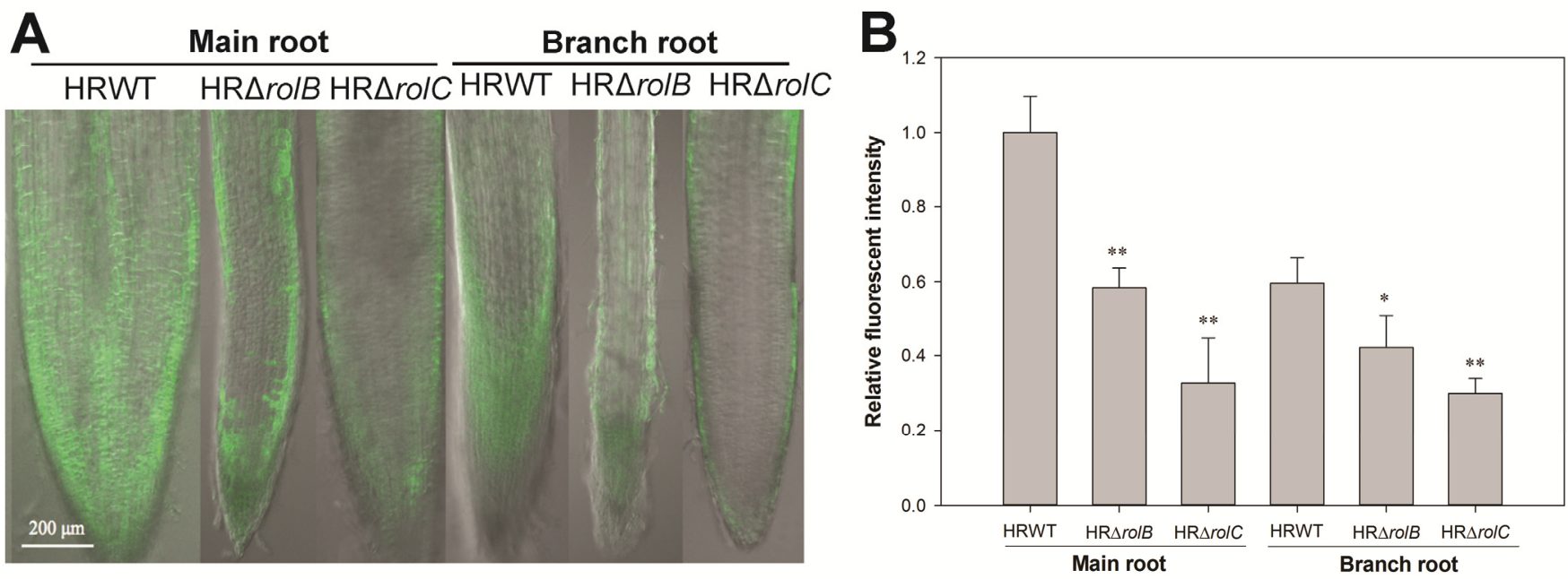

Fig. 4. Reactive oxygen species (ROS) content of HRWT, HR $\Delta$ rolB, and HR $\Delta$ rolC. A, The ROS content was determined using chloromethyl-2',7'-dichlorodihydrofluorescein diacetate, and the fluorescence was observed under a confocal microscope. B, Fluorescent signals were quantified in three biological replicates using ImageJ. The fluorescence intensities of the main roots and branch roots were compared with those of HRWT. $*$ indicates $P<0.05$ and $* *$ indicates $P<0.01)$. 
entiation and thus generate branch roots. In contrast, HR $\Delta$ rolC was significantly different from HRWT in terms of both main root growth and branch root growth. However, HR $\Delta$ rolB and $\mathrm{HR} \Delta$ rolC had similar growth characteristics, and microarray analysis revealed that both mutants had fewer ROS generationrelated transcripts than HRWT, suggesting that rolB and rolC have a similar effect on ROS promotion and similar biological functions. Therefore, we hypothesize that $\operatorname{rolB}$ and $\operatorname{rol} C$ increase cellular ROS levels to promote branch root growth, which is adapted to hairy root growth needs.

Aside from LTP and ROS, other gene sets showing lower expression levels in HR $\Delta$ rolB and HR $\Delta$ rolC compared with HRWT were also enriched in GO terms, including "macromolecular localization," "hydrolase activity," "endomembrane system," "carbohydrate metabolic process," and "ion binding" (Tables 1 and 2). In this article, we discuss LTP and ROS because they were the dominant gene sets and they have not been reported to be related to hairy root-growth regulation. The relationships between hairy root growth and the functions of other genes will be further studied.

None of the hairy root architecture parameters measured in this study showed a normal distribution. We hypothesized that this was due to two factors: i) different T-DNA integration sites and ii) different T-DNA copy numbers. These factors determine the level of expression of the T-DNA genes and the plant genes around the T-DNA. To eliminate these effects, we performed microarray analyses on two different batches, each consisting of a different pool of 24 hairy root clones. From the two-batch microarray, only approximately $20 \%$ of the transcripts with a more than twofold difference were found in both microarray replicates, and we are confident that these genes are regulated by the rol genes, although the T-DNA insertion sites and copy numbers may differ.

Some authors have reported that cell suspension cultures expressing either rolB or rolC driven by the CaMV35S promoter show reduced cellular ROS levels compared with that in untransformed controls (Bulgakov et al. 2008, 2012). In contrast, we found that hairy roots lacking $\mathrm{rolB}$ or $\mathrm{rolC}$ had lower ROS levels, as compared with HRWT (Fig. 4). Our data, therefore, suggest that $\mathrm{rolB}$ and $\mathrm{rolC}$ increase cellular ROS levels in hairy roots. The difference between these results may be explained by the different tissue types in which ROS levels were measured or the different promoters used in each experiment. These data suggest that $\mathrm{rolB}$ and $\mathrm{rolC}$ increase ROS levels when driven by their own promoters in hairy root tissue but decrease ROS levels when driven by the CaMV35S promoter in cell-suspension cultures.

Hairy roots are very useful for studying root biology and for the production of plant secondary metabolites for the pharmaceutical industry. An understanding of how Agrobacterium tumefaciens initiates and prolongs hairy root growth in host plants is a key step toward expanding the application of hairy root technology to other plant species. Although T-DNA transformation can be achieved using A. tumefaciens in some monocots, the plants cannot produce hairy roots. We hypothesize that hairy root generation is the result of T-DNA-dependent reprogramming of the infected cell. In this study, we used tobacco, which can be infected and grows hairy roots easily, to understand how the plant participates in hairy root formation. The finding that either $\mathrm{rolB}$ or $\mathrm{rolC}$ alone can induce hairy root formation indicates that hairy root-formation mechanisms are tightly linked with the cellular functions of RolB and RolC (Spena et al. 1987). By understanding the biochemical and molecular functions of RolB and RolC in hairy roots, we might expand the application of hairy root technology to plants that are resistant to $A$. rhizogenes infection. In this study, we found that hairy roots lost their indefinite growth ability when
rolB or rolC was knocked out. We conclude that $\mathrm{rolB}$ and $\mathrm{rolC}$ play important roles in maintaining root meristem activity. This is a promising result because the means by which the plant retains root meristem activity might be revealed by timecourse assays of differential gene expression in HRWT, $\mathrm{HR} \Delta \mathrm{rolB}$, and $\mathrm{HR} \Delta$ rolC hairy roots.

\section{MATERIALS AND METHODS}

\section{Plant growth and hairy root induction.}

Nicotiana tabacum L. cv. Wisconsin 38 seeds were surfacesterilized using sodium hypochlorite solution (1\% available chlorine) and deposited on culture plates containing solid halfstrength Murashige and Skoog (MS) medium (Caisson Laboratories, Logan, UT, U.S.A.) with $3 \%$ sucrose and $0.3 \%$ (wt/vol) Gelzen (Caisson Laboratories). The tobacco seeds were cultured at $22^{\circ} \mathrm{C}$ with $14 \mathrm{~h}$ of light $\left(100 \mu \mathrm{mol} \mathrm{m}^{-2} \mathrm{~s}^{-1}\right)$.

To induce hairy roots, A. rhizogenes and its mutants were grown in YEB medium at $26^{\circ} \mathrm{C}$ with shaking at $130 \mathrm{rpm}$ for 48 $\mathrm{h}$. The culture was diluted to an optical density at $600 \mathrm{~nm}$ of 0.1 in half-strength MS medium to make the infection liquid. Eight-week-old tobacco leaves were cut into $1-\mathrm{cm}^{2}$ discs and were injected with $0.4 \mu \mathrm{l}$ of the infection liquid. The leaf discs were then planted on solid culture plates at $22^{\circ} \mathrm{C}$ for 3 days in the dark. The discs were subsequently transferred to sterile plates containing solid culture medium with cefotaxime at 300 mg per liter (Caisson Laboratories) to remove A. rhizogenes. At $21 \mathrm{dpi}$, a single root from each leaf disc was transferred onto a fresh sterile plate, and the individual hairy root clone was subcultured every two weeks.

\section{Generation of rol gene knockout strains of $A$. rhizogenes.}

To generate rol-deficient mutants, the $s a c B$-based gene knockout strategy was applied using the $\mathrm{pK} 18 \mathrm{mobsacB}$ vector (Schaefer et al. 1994). Splicing by overlap extension (SOEing) PCR was performed to amplify approximately $1 \mathrm{~kb}$ of sequence upstream and $1 \mathrm{~kb}$ of sequence downstream of the rol gene fragment targeted for deletion by PCR, and the PCR products were subcloned into the $\mathrm{pK} 18$ mobsacB vector. The resulting plasmids were individually transformed into $A$. rhizogenes, which was plated onto YEB agar plates containing kanamycin at $100 \mathrm{ppm}$. To select a mutant, a colony was inoculated into 3 $\mathrm{ml}$ of YEB medium and was incubated with shaking overnight and the culture was spread onto YEB agar plates containing $10 \%$ sucrose. The primers used to generate mutants are listed in Supplementary Table S2.

\section{Statistical analysis.}

The statistical analysis was performed with the R program, version 2.14.1 (R Development Core Team 2011). The DREPI and $\mathrm{R} / \mathrm{L}$ ratio were first analyzed by the Shapiro-Wilk normality test, but neither was normally distributed at the significance level $\alpha=0.05$; thus, nonparametric methods were used. The significance of the group effect was tested by the Kruskal Wallis method and Mann-Whitney U tests with post hoc Bonferroni correction. Hairy root architecture parameters (MRL, BRN, TBRL, and BRD) were statistically analyzed by permutation tests using ANOVA with 1,000 replications. The permutation test was performed as follows: an $\mathrm{F}$ value was obtained using ANOVA to compare one of the rol gene-deficient hairy roots with WTA4-induced hairy roots. The groupings of all the observations were then permuted, and the permuted data were analyzed by ANOVA to obtain another $F$ value. The permutation tests were performed 1,000 times, generating 1,000 F values. The percentage of $F$ values equal to or larger than the original value was reported as the permuted $P$ value; smaller $P$ values indicated more highly significant differences between groups. 
RNA extraction for microarray assay.

Each hairy root clone was cut $1.5 \mathrm{~cm}$ from the root tip, and the root tip was grown in a $250-\mathrm{ml}$ flask containing $50 \mathrm{ml}$ of half-strength liquid MS medium containing 3\% sucrose. The 14-day-old tissue was frozen by liquid nitrogen and was ground into a powder in a mortar. Before thawing, $1 \mathrm{ml}$ of TRIzol reagent (Life Technology, Grand Island, NY, U.S.A.) per $100 \mathrm{mg}$ of tissue powder was added to the mortar to cover the sample. Samples from 24 independent clones of each hairy root group in TRIzol reagent were then pooled to create a sample mixture $(50 \mu \mathrm{l}$ each). Total RNA was extracted with a Direct-zol RNA MiniPrep kit (Zymo Research, Irvine, CA, U.S.A.) following the manufacturer's instructions. RNA from a fast-growing hairy root clone was extracted at 14 days using the same procedure, and RNA from an intact tobacco root was extracted and pooled with 24 root samples obtained at 1-h intervals to avoid circadian variation. The RNA quality was determined using an Agilent 2100 bioanalyzer (Agilent Technologies, Santa Clara, CA, U.S.A.).

\section{Microarray assay and gene ontology.}

A $0.2-\mu \mathrm{g}$ sample of total RNA was amplified using a LowInput Quick-Amp labeling kit (Agilent Technologies) and was labeled with $\mathrm{Cy} 3$ or Cy5 (CyDye; Agilent Technologies) during in vitro transcription. The Cy-labeled cRNAs were hybridized to a dual-channel Agilent Tobacco Oligo $4 \times 44 \mathrm{~K}$ microarray (Agilent Technologies) that was scanned with an Agilent microarray scanner (Agilent Technologies) at $535 \mathrm{~nm}$ for Cy3 and $625 \mathrm{~nm}$ for Cy5. The images were analyzed and normalized by the rank consistency-filtering LOWESS (locally weighted linear regression) method with Feature Extraction Software (version 10.5.1.1; Agilent Technologies). The results were analyzed with GeneSpring GX software (Agilent Technologies), and the differentially expressed genes $(P$ value $<0.05)$ were extracted and sorted using the GO Analysis Toolkit and Database for the Agricultural Community (AgriGO) (Du et al. 2010). The GO enrichment analyses were performed by singular enrichment analysis, using AgriGO with the default settings.

\section{qRT-PCR.}

Primers were designed using Beacon Designer Software with the sequences from the National Center for Biotechnology Information database (listed in Supplementary Table S3). RNA was extracted from 14-day-old hairy roots grown in liquid culture medium. cDNA was synthesized from $1 \mu \mathrm{g}$ of total RNA with the GoScript Reverse Transcription System (Promega, Madison, WI, U.S.A.) following the manufacturer's protocol. qRT-PCR analyses were performed with a CFX384 real-time PCR detection system and iQ SYBR Green Supermix (BioRad, Hercules, CA, U.S.A.), following the manufacturer's protocol. TAC-9 (tobacco ACTIN-9) and beta-TUBULIN were used as reference genes.

\section{ROS staining.}

Hairy root tissue cultured on a sterile plate for 18 days was transferred to a clean petri dish, was equilibrated with $50 \mathrm{mM}$ phosphate buffer ( $\mathrm{pH} \mathrm{7)}$ for $10 \mathrm{~min}$, and was stained with 10 $\mu \mathrm{M}$ 5-(and-6)-CM- $\mathrm{H}_{2}$ DCFDA (Life Technologies) in $50 \mathrm{mM}$ phosphate buffer ( $\mathrm{pH} \mathrm{7)}$ for $30 \mathrm{~min}$ at room temperature. The stained root was observed with an LSM780 confocal microscope (Zeiss, Jena, Germany), and the fluorescent signals were quantified using ImageJ software.

\section{ACKNOWLEDGMENTS}

We deeply appreciate the excellent technical assistance provided by Technology Commons, College of Life Science, NTU (Taiwan), for confocal laser scanning microscopy and real-time PCR. We also thank the Consulting Center of Statistics and Bioinformatics (CCSB), College of BioResources and Agriculture, NTU (Taiwan) for providing experts for consultation. We thank C.-C. Yang for the helpful advice on manuscript preparation. This work was funded by a grant from the National Science Council (number 99-2313-B-002-034-MY3), Taiwan.

\section{LITERATURE CITED}

Baque, M. A., Moh, S. H., Lee, E. J., Zhong, J. J., and Paek, K. Y. 2011. Production of biomass and useful compounds from adventitious roots of high-value added medicinal plants using bioreactor. Biotechnol. Adv. 30:1255-1267.

Batra, J., Dutta, A., Singh, D., Kumar, S., and Sen, J. 2004. Growth and terpenoid indole alkaloid production in Catharanthus roseus hairy root clones in relation to left- and right-termini-linked Ri T-DNA gene integration. Plant Cell Rep. 23:148-154.

Bhattacharjee, S. 2012. The language of reactive oxygen species signaling in plants. J. Bot. doi:10.1155/2012/985298. Published online.

Boisson-Dernier, A., Chabaud, M., Garcia, F., Becard, G., Rosenberg, C., and Barker, D. G. 2001. Agrobacterium rhizogenes-transformed roots of Medicago truncatula for the study of nitrogen-fixing and endomycorrhizal symbiotic associations. Mol. Plant-Microbe Interact. 14:695700 .

Bulgakov, V. P. 2008. Functions of rol genes in plant secondary metabolism. Biotechnol. Adv. 26:318-324.

Bulgakov, V. P., Aminin, D. L., Shkryl, Y. N., Gorpenchenko, T. Y., Veremeichik, G. N., Dmitrenok, P. S., and Zhuravlev, Y. N. 2008. Suppression of reactive oxygen species and enhanced stress tolerance in Rubia cordifolia cells expressing the rolC oncogene. Mol. PlantMicrobe Interact. 21:1561-1570.

Bulgakov, V. P., Gorpenchenko, T. Y., Veremeichik, G. N., Shkryl, Y. N., Tchernoded, G. K., Bulgakov, D. V., Aminin, D. L., and Zhuravlev, Y. N. 2012. The rolB gene suppresses reactive oxygen species in transformed plant cells through the sustained activation of antioxidant defense. Plant Physiol. 158:1371-1381.

Capone, I., Cardarelli, M., Mariotti, D., Pomponi, M., Depaolis, A., and Costantino, P. 1991. Different promoter regions control level and tissuespecificity of expression of Agrobacterium rhizogenes- rolB gene in plants. Plant Mol. Biol. 16:427-436.

Casanova, E., Trillas, M. I., Moysset, L., and Vainstein, A. 2005. Influence of rol genes in floriculture. Biotechnol. Adv. 23:3-39.

DeBoer, K. D., Dalton, H. L., Edward, F. J., and Hamill, J. D. 2011. RNAi-mediated down-regulation of ornithine decarboxylase (ODC) leads to reduced nicotine and increased anatabine levels in transgenic Nicotiana tabacum L. Phytochemistry 72:344-355.

DePaolis, A., Sabatini, S., DePascalis, L., Costantino, P., and Capone, I. 1996. A rolB regulatory factor belongs to a new class of single zinc finger plant proteins. Plant J. 10:215-223.

Du, Z., Zhou, X., Ling, Y., Zhang, Z., and Su, Z. 2010. agriGO: A GO analysis toolkit for the agricultural community. Nucl. Acids Res. 38:6470 .

Estruch, J. J., Chriqui, D., Grossmann, K., Schell, J., and Spena, A. 1991. The plant oncogene rolC is responsible for the release of cytokinins from glucoside conjugates. EMBO (Eur. Mol. Biol. Organ.) J. 10:28892895.

Faiss, M., Strnad, M., Redig, P., Dolezal, K., Hanus, J., VanOnckelen, H., and Schmulling, T. 1996. Chemically induced expression of the rolCencoded $\beta$-glucosidase in transgenic tobacco plants and analysis of cytokinin metabolism: rolC does not hydrolyze endogenous cytokinin glucosides in planta. Plant J. 10:33-46.

Filetici, P., Moretti, F., Camilloni, G., and Mauro, M. L. 1997. Specific interaction between a Nicotiana tabacum nuclear protein and the Agrobacterium rhizogenes rolB promoter. J. Plant Physiol. 151:159-165.

Filippini, F., Rossi, V., Marin, O., Trovato, M., Costantino, P., Downey, P. M., Lo Schiavo, F., and Terzi, M. 1996. A plant oncogene as a phosphatase. Nature 379:499-500.

Hamill, J. D., Parr, A. J., Robins, R. J., and Rhodes, M. J. C. 1986. Secondary product formation by cultures of Beta vulgaris and Nicotiana rustica transformed with Agrobacterium rhizogenes. Plant Cell Rep. 5:111-114.

Kader, J. C. 1996. Lipid-transfer proteins in plants. Annu. Rev. Plant Physiol. Plant Mol. Biol. 47:627-654.

Lee, K. T., Chen, S. C., Chiang, B. L., and Yamakawa, T. 2007. Heatinducible production of $\beta$-glucuronidase in tobacco hairy root cultures. Appl. Microbiol. Biotechnol. 73:1047-1053.

Levesque, H., Delepelaire, P., Rouze, P., Slightom, J., and Tepfer, D. 1988. Common evolutionary origin of the central portions of the $\mathrm{Ri} \mathrm{T}_{\mathrm{L}}$-DNA of Agrobacterium rhizogenes and the Ti T-DNA of Agrobacterium 
tumefaciens. Plant Mol. Biol. 11:731-744.

Maurel, C., Leblanc, N., Barbier-Brygoo, H., Perrot-Rechenmann, C., Bouvier-Durand, M., and Guern, J. 1994. Alterations of auxin perception in rolB-transformed tobacco protoplasts. Time course of $\mathrm{rolB}$ mRNA expression and increase in auxin sensitivity reveal multiple control by auxin. Plant Physiol. 105:1209-1215.

Moriuchi, H., Okamoto, C., Nishihama, R., Yamashita, I., Machida, Y., and Tanaka, N. 2004. Nuclear localization and interaction of RolB with plant 14-3-3 proteins correlates with induction of adventitious roots by the oncogene rolB. Plant J. 38:260-275.

Nilsson, O., Moritz, T., Sundberg, B., Sandberg, G., and Olsson, O. 1996. Expression of the Agrobacterium rhizogenes rolC gene in a deciduous forest tree alters growth and development and leads to stem fasciation. Plant Physiol. 112:493-502.

Ono, N. N., and Tian, L. 2011. The multiplicity of hairy root cultures: Prolific possibilities. Plant Sci. 180:439-446.

R Development Core Team. 2011, R: A Language and Environment for Statistical Computing. The R Foundation for Statistical Computing, Vienna, Austria.

Rigden, D. J., and Carneiro, M. 1999. A structural model for the rolA protein and its interaction with DNA. Proteins 37:697-708.

Schaefer, A., Tauch, A., Jager, W., Kalinowski, J., Thierbach, G., and Puhler, A. 1994. Small mobilizable multi-purpose cloning vectors derived from the Escherichia coli plasmids pK18 and pK19: Selection of defined deletions in the chromosome of Corynebacterium glutamicum. Gene 145:69-73.

Schmulling, T., Schell, J., and Spena, A. 1988. Single genes from Agrobacterium rhizogenes influence plant development. EMBO (Eur. Mol. Biol. Organ.) J. 7:2621-2629.

Shoji, T., Kajikawa, M., and Hashimoto, T. 2010. Clustered transcription factor genes regulate nicotine biosynthesis in tobacco. Plant Cell 22:3390-3409.

Spena, A., Schmulling, T., Koncz, C., and Schell, J. S. 1987. Independent and synergistic activity of $\operatorname{rol} A, B$ and $C$ loci in stimulating abnormal growth in plants. EMBO (Eur. Mol. Biol. Organ.) J. 6:3891-3899.

Sun, L. Y., Monneuse, M. O., Martin-Tanguy, J., and Tepfer, D. 1991.
Changes in flowering and the accumulation of polyamines and hydroxycinnamic acid-polyamine conjugates in tobacco plants transformed by the rolA locus from the $\mathrm{Ri}_{\mathrm{L}}$-DNA of Agrobacterium rhizogenes. Plant Sci. 80:145-156

Swain, S. S., Sahu, L., Barik, D. P., and Chand, P. K. 2010. Agrobacterium $\times$ plant factors influencing transformation of 'Joseph's coat' (Amaranthus tricolor L.). Sci. Hortic. 125:461-468.

Thoma, S., Hecht, U., Kippers, A., Botella, J., Devries, S., and Somerville, C. 1994. Tissue-specific expression of a gene encoding a cell wall-localized lipid transfer protein from Arabidopsis. Plant Physiol. 105:35-45.

Trovato, M., Maras, B., Linhares, F., and Costantino, P. 2001. The plant oncogene rolD encodes a functional ornithine cyclodeaminase. Proc. Natl. Acad. Sci. U.S.A. 98:13449-13453.

Tsukagoshi, H., Busch, W., and Benfey, P. N. 2010. Transcriptional regulation of ROS controls transition from proliferation to differentiation in the root. Cell 143:606-616.

Vilaine, F., Rembur, J., Chriqui, D., and Tepfer, M. 1998. Modified development in transgenic tobacco plants expressing a rolA:: gus translational fusion and subcellular localization of the fusion protein. Mol. PlantMicrobe Interact. 11:855-859.

White, F. F., Taylor, B. H., Huffman, G. A., Gordon, M. P., and Nester, E. W. 1985. Molecular and genetic analysis of the transferred DNA regions of the root-inducing plasmid of Agrobacterium rhizogenes. J. Bacteriol. 164:33-44.

Yokoyama, R., Hirose, T., Fujii, N., Aspuria, E. T., Kato, A., and Uchimiya, H. 1994. The rolC promoter of Agrobacterium rhizogenes $\mathrm{Ri}$ plasmid is activated by sucrose in transgenic tobacco plants. Mol. Gen. Genet. 244:15-22.

Zhou, M. L., Zhu, X. M., Shao, J. R., Tang, Y. X., and Wu, Y. M. 2011. Production and metabolic engineering of bioactive substances in plant hairy root culture. Appl. Microbiol. Biotechnol. 90:1229-1239.

\section{AUTHOR-RECOMMENDED INTERNET RESOURCE}

AgriGO's analysis toolkit and database: bioinfo.cau.edu.cn/agriGO 